\section{CONSUMO DE FRUTAS, VERDURAS E LEGUMES POR GESTANTES ADOLESCENTES}

\author{
Consumption of fruits, green vegetables and legumes by \\ pregnant adolescents
}

\author{
Consumo de frutas, verduras y legumbres de adolescentes \\ embarazadas
}

\section{RESUMO}

Objetivo: Determinar o estado nutricional e os fatores associados ao consumo alimentar de frutas, verduras e legumes por gestantes adolescentes atendidas em um serviço público de referência para assistência pré-natal. Métodos: Estudo transversal e analítico, com 73 gestantes adolescentes de 10 a 19 anos, atendidas no Núcleo de Assistência ao Adolescente (NASA) do Hospital Materno Infantil, em São Luís, Maranhão. Utilizou-se o Questionário de Frequência de Consumo Alimentar (QFCA), medidas antropométricas (peso, altura, índice de massa corporal - IMC - pré-gravídico e gravídico) e questionário socioeconômico. As variáveis dependentes foram o consumo de frutas, verduras e legumes, e as independentes foram escolaridade, estado civil, raça, renda, situação demográfica, dados gestacionais e antropométricos. Resultados: Observou-se que 39,7\% apresentaram IMC pré-gestacional de desnutrição, $50,7 \%$ de eutrofia, e menos de $10 \%$ sobrepeso ou obesidade. Para o IMC gestacional, os valores se alteraram, com $27,4 \%$ das gestantes desnutridas, $57,5 \%$ eutróficas e $15,1 \%$ com sobrepeso. Observou-se que os maiores percentuais de adequação para o consumo de frutas, verduras e legumes foram em adolescentes casadas ou em união estável $(65,4 \%)$, que não trabalhavam $(92,3 \%)$ e com renda familiar menor que 1 salário mínimo (84,62\%). Entretanto, a única associação positiva encontrada com o consumo de frutas, verduras e legumes foi o início do pré-natal. Conclusão: A maior parte das gestantes avaliadas apresentou-se eutrófica, apesar de cerca de um quarto apresentar baixo peso durante a gestação. Além disso, elas não consumiam uma dieta balanceada, com uma ingestão abaixo do recomendado de FVL. Entre os fatores relacionados a um melhor consumo de FVL destaca-se o início do acompanhamento pré-natal no primeiro trimestre.

Descritores: Adolescente; Gestantes; Consumo de Alimentos.

\section{ABSTRACT}

Objective: To determine the nutritional status and the factors associated with dietary intake of fruits, green vegetables and legumes by pregnant adolescents attending a public service of reference for prenatal care. Methods: Analytical cross-sectional study, with 73 pregnant adolescents aged between 10 and 19 years, attended to at the Service Center for Adolescents (NASA) of the Mother and Child Hospital, in São Luís, Maranhão. The study used the Food Frequency Questionnaire (FFQ), anthropometric measurements (weight, height, pregravid and gestational body mass index), and a socioeconomic questionnaire. The dependent variable was the consumption of fruits, green vegetables and legumes, and the independent variables were level of education, civil status, race, income, demographic status, pregnancy and anthropometric data. Results: It was observed that $39.7 \%$ had pregravid BMI of malnutrition, $50.7 \%$ were eutrophic, and less than $10 \%$ were overweight or obese. For the gestational BMI, the values changed, with $27.4 \%$ of undernourished pregnant women, $57.5 \%$ eutrophic and $15.1 \%$ overweight. The highest percentages of adequacy for consumption of fruits, green vegetables and legumes were observed in adolescents who were married or in a stable union (65.4\%), did not work (92.3\%), and had family income below one minimum wage (84.62\%). However, the only positive association found with the consumption of fruits, green vegetables and legumes was the beginning of prenatal care. Conclusion: The
Artigo Original
1) Universidade Federal do Maranhão UFMA - São Luís (MA) - Brasil

2) Universidade Estadual do Norte Fluminense Darcy Ribeiro - UENF - Rio de Janeiro (RJ) - Brasil
Recebido em: 30/05/2016 Revisado em: 03/08/2016 Aceito em: 28/11/2016 
major part of the evaluated pregnant adolescents was eutrophic, although about one fourth presented low weight during pregnancy. Moreover, they did not consume a balanced diet, with an intake of fruits, green vegetables and legumes below the recommended. Among the factors related to better consumption of these foods, the beginning of prenatal follow-up in the first trimester stands out.

Descriptors: Adolescents; Pregnant women; Food Consumption.

\section{RESUMEN}

Objetivo: Determinar el estado nutricional y los factores asociados al consumo alimentario de frutas, verduras y legumbres por adolescentes embarazadas asistidas en un servicio público de referencia para asistencia prenatal. Métodos: Estudio transversal y analitico con 73 embarazadas adolescentes entre 10 y 19 años asistidas en el Núcleo de Asistencia al Adolescente (NASA) del Hospital Materno Infantil de São Luís, Maranhão. Se utilizó el Cuestionario de Frecuencia de Consumo Alimentario (QFCA), las medidas antropométricas (peso, altura, índice de masa corporal IMC - pre-gravidico y gravidico) y cuestionario socioeconómico. Las variables dependientes fueron el consumo de frutas, verduras y legumbres y las independientes fueron la escolaridad, el estado civil, la raza, la renta, la situación demográfica, los datos de la gestación y antropométricos. Resultados: Se observó que el 39,7\% presentaron IMC pre-gestacional de desnutrición, el $50,7 \%$ de eutrofia y menos del $10 \%$ de sobrepeso u obesidad. Los valores se alteraron para el IMC gestacional con el 27,4\% de las embarazadas desnutridas, el 57,5\% de eutróficas y el $15,1 \%$ de sobrepeso. Se observó que los mayores porcentuales de adecuación para el consumo de frutas, verduras y legumbres fueron en adolescentes casadas o con pareja de hecho $(65,4 \%)$, que no trabajaban $(92,3 \%)$ y tenian renta familiar menor que 1 sueldo mínimo (84,62\%). Sin embargo, la única asociación positiva encontrada con el consumo de frutas, verduras y legumbres fue el inicio del prenatal. Conclusión: La gran mayoría de las embarazadas evaluadas estaba eutrófica aunque el alrededor de un cuarto de ellas tuvo bajo peso durante el embarazo. Además, ellas no tenían una dieta balanceada con una ingestión abajo del recomendado de FVL. De entre los factores relacionados al mejor consumo de FVL se destaca el inicio del seguimiento del prenatal en el primer trimestre.

Descriptores: Adolescente; Mujeres Embarazadas; Consumo de Alimentos.

\section{INTRODUÇÃO}

A gravidez na adolescência é considerada, em alguns países, problema de saúde pública, uma vez que pode acarretar complicações obstétricas com repercussões para a mãe e o recém-nascido, bem como problemas psicossociais e econômicos. No Brasil, tem sido referido um aumento da incidência da gravidez na adolescência, com uma variação de 14 a $22 \%{ }^{(1)}$.
A taxa de fecundidade no grupo de mulheres entre 10 e 19 anos aumentou consideravelmente nas últimas quatro décadas no Brasil. Em 1980, a fecundidade das mulheres de 15 a 19 anos de idade representava $9,1 \%$ da fecundidade total do país. Em 2000, esse percentual aumentou para 19,4\%. Do total de nascidos vivos do país, foram identificados $0,9 \%$ de nascidos vivos de mães entre 10 e 14 anos e $22,4 \%$ de nascidos vivos de mães entre 15 e 19 anos de idade ${ }^{(2)}$.

Durante a gestação, há um incremento das necessidades nutricionais e, na gestante adolescente, este aumento é ainda maior por que se superpõem as demandas referentes ao crescimento da mãe e aquelas relacionadas ao desenvolvimento do feto ${ }^{(3)}$. Em decorrência disso, o estado nutricional está entre os riscos associados a esse tipo de gravidez $^{(4)}$.

Além das necessidades aumentadas, pesquisas têm documentado que o hábito alimentar dessas gestantes é inadequado. Observa-se uma alta ingestão energética associada à deficiência de nutrientes essenciais ${ }^{(5)}$, como cálcio, ferro, folato, zinco e fibras, e um excessivo consumo de gorduras $^{(6)}$, sendo orientada por escolha de alimentos mais atrativos, disponíveis, práticos e baratos ${ }^{(5,7)}$.

Vários estudos epidemiológicos têm sugerido a importância do consumo de frutas e hortaliças na promoção da saúde e prevenção de doenças crônicas não transmissíveis, como doenças cardiovasculares, câncer, diabetes e obesidade ${ }^{(8)}$. No entanto, apenas uma minoria da população atinge a recomendação de consumo diário de frutas e vegetais (mínimo de 400 gramas por dia), sobretudo indivíduos mais jovens, entre os quais o consumo é ainda menor $^{(9,10)}$.

Portanto, a ingestão adequada dos diferentes componentes da dieta irá garantir um desenvolvimento saudável para a mãe e para o feto. Se a ingestão dietética for insuficiente e se os estoques de nutrientes da gestante estiverem baixos, o feto recorrerá às reservas préconcepcionais para se suprir, ocasionando comprometimento do binômio materno-fetal ${ }^{(11)}$.

O comportamento alimentar da gestante adolescente tende a sofrer poucas modificações quando comparado ao período que antecedeu a gravidez. A falta de conhecimento sobre alimentação saudável pelas jovens grávidas refletese nas suas escolhas alimentares, que são influenciadas por fatores como a fome, o desejo, o paladar aumentado, a conveniência, a disponibilidade do alimento e as influências culturais e familiares $^{(3)}$.

O tema alimentação de gestante adolescente tem sido pouco explorado, com isso, há escassez de pesquisas sobre este assunto na literatura, e a maioria delas foi realizada nos Estados Unidos. Conhecer o consumo habitual dos alimentos por este grupo de mulheres poderá servir para orientar 
os programas de controle de ganho de peso gestacional e a promoção da alimentação saudável entre estas jovens, além de gerar qualidade de vida para a gestante e o recémnascido. Diante do exposto, este estudo teve como objetivo determinar o estado nutricional e os fatores associados ao consumo alimentar de frutas, verduras e legumes em gestantes adolescentes atendidas em um serviço público de referência para assistência pré-natal.

\section{MÉTODOS}

Trata-se de um estudo transversal e analítico realizado com gestantes adolescentes, com idades entre 10 e 19 anos, atendidas no Núcleo de Assistência ao Adolescente (NASA) do Hospital Materno Infantil da cidade de São Luís, Maranhão, no período de setembro a dezembro de 2015.

Fizeram parte deste estudo as gestantes com até 19 anos de idade, com menarca maior que dois anos e que aceitaram participar do estudo mediante assinatura do Termo de Assentimento e da assinatura do Termo de Consentimento Livre e Esclarecido (TCLE) pelos responsáveis.

Não foram incluídas na amostra pacientes com necessidades especiais que inviabilizassem a compreensão e preenchimento dos questionários ou a aferição das medidas antropométricas, nem aquelas que não residiam no município de São Luís - MA. Desta forma, a seleção da amostra se deu por conveniência e totalizou 73 pacientes.

Inicialmente, foi aplicado um questionário que continha questões com dados demográficos e gestacionais, como data de nascimento, escolaridade ( $>$ 8anos de estudos e $\leq 8$ anos de estudos) e data de início do pré-natal $\left(1^{\circ}, 2^{\circ}\right.$ e $3^{\circ}$ trimestre). O questionário trazia ainda questões para a classificação socioeconômica (raça, estado civil, ocupação, renda e religião). Para a uniformização da técnica de aplicação do instrumento, as questões foram lidas pelo pesquisador para todas as pacientes na sala de espera para consulta com o obstetra, independentemente do grau de escolaridade.

Para avaliar o consumo alimentar, foi utilizado um Questionário de Frequência de Consumo Alimentar (QFCA), previamente validado, composto por uma lista de 24 alimentos, semiquantitativo, no qual constavam porções padronizadas para cada item, por exemplo: para arroz, a porção padrão era " 3 colheres de servir cheia"; para macarrão, "um pegador"; para farinha de mandioca, "uma colher de sopa"; para pão, "um pão francês"; para feijão, "uma concha". Desse questionário, constavam as seguintes opções de frequência de consumo: mais de 3 vezes por dia, de 2 a 3 vezes por dia, uma vez por dia, de 5 a 6 vezes por semana, de 2 a 4 vezes por semana, uma vez por semana,
1 a 3 vezes por mês, e nunca ou quase nunca antes da gestação $^{(12)}$.

A lista de alimentos do QFCA foi obtida com base em questionários utilizados em investigação do consumo de alimentos de base populacional ${ }^{(12)}$. A essa lista foi adicionada a farinha de mandioca, cujo consumo é considerado frequente entre adolescentes no município de São Luís. Na fase de validação do questionário, observouse, pelo consumo regional, que somente as frutas banana e laranja foram relatadas pelas adolescentes. Portanto, apenas essas duas frutas foram incluídas no questionário. $\mathrm{O}$ consumo de frutas, verduras e legumes foi considerado adequado quando atingia a recomendação de 4 a 5 porções diárias $^{(13)}$.

Para avaliar o estado nutricional, foram aferidos o peso e a altura corporais atuais durante a consulta e peso pré-gravídico, autoreferido ou o mesmo apresentado no primeiro trimestre, conforme recomendação ${ }^{(14)}$. Para mensuração do peso, utilizou-se balança eletrônica digital portátil, tipo plataforma, marca $\mathrm{Kratos}^{\circledR}$, com capacidade para 150 quilogramas e sensibilidade de 50 gramas. A estatura foi aferida utilizando o antropômetro Alturaexata ${ }^{\circledR}$, de altura máxima de 2,13 metros e precisão de 1 milímetro. Foi identificado o estado nutricional pré-gestacional através do IMC pré-gestacional, dividindo-se o peso $(\mathrm{kg})$ pela altura ao quadrado $\left(\mathrm{m}^{2}\right)$.

O IMC pré-gestacional foi classificado de acordo com os seguintes pontos de corte: baixo peso (IMC $<19,8 \mathrm{~kg} / \mathrm{m}^{2}$ ), eutrofia (IMC 19,8-26 kg/m²), sobrepeso (IMC 26 - 29kg/ $\mathrm{m}^{2}$ ) e obesidade $\left(\mathrm{IMC}>29 \mathrm{~kg} / \mathrm{m}^{2}\right)^{(15)}$.

Para avaliação nutricional atual das gestantes, foi utilizado o gráfico adotado pelo Ministério da Saúde ${ }^{(9)}$, que utiliza o IMC atual e a idade gestacional. Obtendo os pontos de corte: baixo peso, eutrofia, sobrepeso e obesidade. A idade gestacional foi calculada a partir do último dia da menstruação das gestantes ${ }^{(16)}$.

A normalidade das variáveis quantitativas foi analisada pelo teste Shapiro Wilk. Os dados foram apresentados por meio de média e desvio padrão (média \pm DP) para as variáveis quantitativas, e, as qualitativas, por frequência e porcentagem. Para identificação dos fatores associados ao consumo de frutas, verduras e legumes foi utilizado o modelo de regressão de Poisson. O nível de significância adotado foi de $5 \%$, entretanto, as variáveis independentes que apresentaram p-valor menor que 0,20 foram consideradas no modelo de regressão multivariado. A seleção das variáveis foi realizada pelo método passo a passo (stepwise) por eliminação e foi utilizado o nível de significância de $5 \%$. Os dados foram analisados no programa estatístico STATA 12.0 
Este estudo foi aprovado pelo comitê de ética de pesquisa do Hospital Universitário da Universidade Federal do Maranhão sob o parecer $\mathrm{n}^{\circ}$. 1.175.244, de acordo com a resolução do Conselho Nacional de Saúde nº. 466/2012.

\section{RESULTADOS}

Foram avaliadas 73 gestantes adolescentes, com idade média de $16,7 \pm 1,3$ anos. A maioria tinha mais de oito anos de estudo $(74 \%, \mathrm{n}=54)$, era da raça negra/ parda $(82,2 \%, \mathrm{n}=$ $60)$, casada/união estável $(53,4 \%, n=39)$. A maior parcela das gestantes adolescentes não trabalhava $(94,5 \%, n=69)$, possuíam renda familiar de um a três salários mínimos $(71,2 \%, \mathrm{n}=52)$, com 4 ou mais moradores no domicílio $(60,3 \%, n=44)$ (Tabela I).

Quando avaliado o estado nutricional anterior à gestação, de acordo com o IMC pré-gestacional, 49,3\% $(n=36)$ apresentou classificação de risco nutricional (desnutrição, sobrepeso e obesidade). Destas, o percentual mais expressivo foi apresentado para desnutrição $(39,7 \%$, $\mathrm{n}=29$ ).

Quando avaliado o IMC de acordo com a idade gestacional, um percentual menos expressivo para risco nutricional foi encontrado $(42,5 \%, \mathrm{n}=31)$ quando comparado ao IMC pré-gestacional. Os valores para desnutrição diminuíram para $27,4 \%(\mathrm{n}=20)$. O número de gestantes eutróficas aumentou para $57,5 \%(\mathrm{n}=42)$, assim como o de gestantes com sobrepeso $(15,1 \%, \mathrm{n}=11)$. Além disto, o quadro de obesidade foi inexistente. Isso demonstra que mais importante, que o estado nutricional anterior à gestação, é o ganho de peso gestacional.

Verificou-se que $35,6 \% \quad(n=26) \quad$ das gestantes apresentaram um consumo adequado de FVL (Tabela I) e entre os alimentos consumidos, em maior frequência diária, estão os cereais (arroz e pão), o leite e a margarina. O açúcar e a banana também aparecem com uma frequência de

Tabela I - Características socioeconômicas, demográficas e de consumo de frutas, verduras e legumes das gestantes adolescentes atendidas em um serviço público de referência pré-natal. São Luís, Maranhão, 2015.

\begin{tabular}{|c|c|c|}
\hline Variáveis & Número de Gestantes & $\%$ \\
\hline \multicolumn{3}{|l|}{ Escolaridade } \\
\hline$\leq 8$ anos & 19 & 26 \\
\hline$>8$ anos & 54 & 74 \\
\hline \multicolumn{3}{|l|}{ Estado civil } \\
\hline Solteira & 34 & 46,6 \\
\hline Casada/ União estável & 39 & 53,4 \\
\hline \multicolumn{3}{|l|}{ Raça } \\
\hline Branca & 13 & 17,8 \\
\hline Negra/parda & 60 & 82,2 \\
\hline \multicolumn{3}{|c|}{ Número de moradores no domicílio } \\
\hline 1 & 2 & 2,7 \\
\hline 2 a 3 & 26 & 35,6 \\
\hline 4 ou mais & 44 & 60,3 \\
\hline \multicolumn{3}{|l|}{ Gestante trabalha } \\
\hline Sim & 4 & 5,5 \\
\hline Não & 69 & 94,5 \\
\hline \multicolumn{3}{|l|}{ Religião } \\
\hline Católica & 39 & 53,2 \\
\hline Protestante & 18 & 24,7 \\
\hline Outras & 14 & 19,2 \\
\hline \multicolumn{3}{|l|}{ Renda } \\
\hline$<$ Salário mínimo & 13 & 17,8 \\
\hline 1 a 3 salários mínimos & 52 & 71,2 \\
\hline > 3 salários mínimos & 8 & 11,0 \\
\hline \multicolumn{3}{|c|}{ Consumo de frutas, verduras e legumes } \\
\hline Adequado & 26 & 35,6 \\
\hline Inadequado & 47 & 64,4 \\
\hline
\end{tabular}


Tabela II - Prevalência diária e semanal do consumo de alimentos pelas adolescentes gestantes atendidas em um serviço público de referência pré-natal. São Luís, Maranhão, 2015.

\begin{tabular}{lccccc}
\hline Alimentos & $\begin{array}{c}\text { Diário } \\
\mathbf{n}(\mathbf{\%})\end{array}$ & $\begin{array}{c}\mathbf{5} \mathbf{a} \mathbf{6} / \mathbf{s} \\
\mathbf{n}(\mathbf{\%})\end{array}$ & $\begin{array}{c}\mathbf{2} \mathbf{a} \mathbf{4} / \mathbf{s} \\
\mathbf{n}(\mathbf{\%})\end{array}$ & $\begin{array}{c}\mathbf{1} / \mathbf{s} \\
\mathbf{n}(\mathbf{\%})\end{array}$ & $\begin{array}{c}\text { Eventualmente } \\
\mathbf{n}(\mathbf{\%})\end{array}$ \\
\hline Arroz & $71(97,3)$ & $2(2,7)$ & $0(0,0)$ & $0(0,0)$ & $0(0,0)$ \\
Açúcar & $66(90,4)$ & $0(0,0)$ & $2(2,7)$ & $0(0,0)$ & $5(6,9)$ \\
Margarina & $62(84,9)$ & $1(1,4)$ & $4(5,5)$ & $1(1,4)$ & $5(6,8)$ \\
Pão & $56(76,7)$ & $4(5,5)$ & $6(8,2)$ & $0(0,0)$ & $0(0,0)$ \\
Leite & $55(71,4)$ & $0(0,0)$ & $8(11,0)$ & $4(5,5)$ & $6(8,2)$ \\
Banana & $31(42,4)$ & $7(9,6)$ & $18(24,7)$ & $6(8,2)$ & $11(15,1)$ \\
Sucos naturais & $27(37,0)$ & $3(4,1)$ & $16(21,9)$ & $7(9,6)$ & $20(27,4)$ \\
Farinha & $26(35,6)$ & $2(2,7)$ & $6(8,2)$ & $7(9,6)$ & $32(43,9)$ \\
Biscoito salgado & $25(34,3)$ & $4(5,5)$ & $15(20,5)$ & $8(11,0)$ & $21(28,7)$ \\
Laranja & $23(31,5)$ & $3(4,1)$ & $21(28,8)$ & $10(13,7)$ & $16(21,9)$ \\
Verduras/legumes & $22(30,2)$ & $8(11,0)$ & $22(30,0)$ & $3(4,1)$ & $18(24,7)$ \\
Feijão & $17(23,3)$ & $4(5,5)$ & $39(53,4)$ & $10(13,7)$ & $3(4,1)$ \\
Refrigerante & $15(20,6)$ & $3(4,1)$ & $18(24,7)$ & $11(15,0)$ & $26(35,6)$ \\
Chocolate & $12(16,5)$ & $1(1,4)$ & $16(21,8)$ & $6(8,2)$ & $38(52,1)$ \\
Ovo & $11(15,3)$ & $2(2,7)$ & $23(32,0)$ & $12(16,7)$ & $24(33,3)$ \\
Salgadinhos & $10(13,7)$ & $1(1,4)$ & $16(22,9)$ & $13(17,8)$ & $33(44,2)$ \\
Carne bovina & $6(8,2)$ & $10(13,7)$ & $46(63,0)$ & $4(5,5)$ & $7(9,6)$ \\
Frango & $5(6,8)$ & $10(13,7)$ & $43(59,0)$ & $6(8,2)$ & $9(12,3)$ \\
Queijo & $5(6,8)$ & $0(0,0)$ & $11(15,1)$ & $14(19,2)$ & $43(58,9)$ \\
Macarrão & $4(5,4)$ & $2(2,7)$ & $28(38,4)$ & $18(24,7)$ & $21(28,8)$ \\
Salsicha/linguiça & $3(4,1)$ & $0(0,0)$ & $9(12,3)$ & $14(19,2)$ & $47(64,4)$ \\
Pizza & $2(2,8)$ & $0(0,0)$ & $5(6,9)$ & $9(12,3)$ & $57(78,0)$ \\
Peixe & $2(2,7)$ & $2(2,7)$ & $24(33,0)$ & $19(26,0)$ & $26(35,6)$ \\
\hline S & & & & & \\
\hline
\end{tabular}

s: semana.

consumo expressiva, principalmente quando comparados às verduras, legumes e frutas, que tiveram um consumo bastante reduzido. O ovo também foi pouco citado, sendo um alimento consumido eventualmente ou 2 a 4 vezes por na semana. Os dados da frequência de ingestão, de alimentos consumidos, estão descritos na tabela II.

Relacionando o consumo alimentar de frutas, verduras e legumes das adolescentes gestantes com as características socioeconômicas encontradas (Tabela III), observou-se que os maiores percentuais de adequação foram em adolescentes com estado civil casada, união estável $65,4 \%(\mathrm{n}=17)$, que não trabalhavam $92,3 \%(\mathrm{n}=24)$ e com renda familiar de menos de 1 salário mínimo 84,62\% (n=22).

Na tabela IV, verifica-se que houve associação positiva entre o início do pré-natal e o consumo de frutas, verduras e legumes, e entre as que fazem o consumo adequado de frutas, verduras e legumes, $69,2 \%(n=51)$ iniciaram o pré-natal no primeiro trimestre, ou seja, as gestantes que começaram o pré-natal mais cedo apresentaram uma melhor ingestão de frutas, verduras e legumes. 
Tabela III - Análise não ajustada da associação entre o consumo de frutas, verduras e legumes e características socioeconômicas de adolescentes gestantes atendidas em um serviço público de referência pré-natal. São Luís, Maranhão, 2015.

\begin{tabular}{|c|c|c|c|c|c|}
\hline \multirow[b]{2}{*}{ Variáveis } & & \multicolumn{2}{|c|}{ Consumo } & \multirow[b]{2}{*}{ RP (IC 95\%) } & \multirow[b]{2}{*}{ p-valor } \\
\hline & & $\begin{array}{l}\text { adequado } \\
\text { n (\%) }\end{array}$ & $\begin{array}{c}\text { inadequado } \\
\mathrm{n}(\%)\end{array}$ & & \\
\hline \multicolumn{5}{|l|}{ Escolaridade } & \multirow[t]{3}{*}{0,127} \\
\hline$>8$ anos estudo & & $16(61,5)$ & $38(80,9)$ & 1,00 & \\
\hline$\leq 8$ anos estudo & & $10(38,5)$ & $9(19,1)$ & $0,67(0,41 ; 1,12)$ & \\
\hline \multicolumn{2}{|l|}{ Estado civil } & & & & \multirow[t]{3}{*}{0,131} \\
\hline \multicolumn{2}{|l|}{ Solteira } & $9(34,6)$ & $25(53,2)$ & $1,30(0,92 ; 1,84)$ & \\
\hline \multicolumn{2}{|l|}{ Casada/ União estável } & $17(65,4)$ & $22(46,8)$ & 1,00 & \\
\hline \multicolumn{2}{|l|}{ Raça } & & & & \multirow{2}{*}{0,130} \\
\hline \multicolumn{2}{|l|}{ Branca } & $7(26,9)$ & $6(12,8)$ & 1,00 & \\
\hline \multicolumn{2}{|l|}{ Negra/parda } & $19(73)$ & $41(87,2)$ & $1,48(0,80 ; 2,74)$ & \multirow{5}{*}{0,546} \\
\hline \multicolumn{2}{|l|}{ Número de moradores no domicílio } & & & & \\
\hline \multicolumn{2}{|l|}{1} & $0(-)$ & $2(4,26)$ & 1,00 & \\
\hline \multicolumn{2}{|l|}{2 a 3} & $9(34,6)$ & $17(36,2)$ & $(0,50 ; 0,87)$ & \\
\hline \multicolumn{2}{|l|}{4 ou mais } & $17(65,4)$ & $27(57,4)$ & $(0,50 ; 0,80)$ & \\
\hline \multicolumn{2}{|l|}{ Gestante trabalha } & & & & \multirow{3}{*}{0,603} \\
\hline \multicolumn{2}{|l|}{ Sim } & $2(7,7)$ & $2(4,3)$ & 1,00 & \\
\hline \multicolumn{2}{|l|}{ Não } & $24(92,3)$ & $45(95,7)$ & $1,30(0,48 ; 3,55)$ & \\
\hline Renda Familiar & & & & & 0,673 \\
\hline$<1$ salário mínimo & & $22(84,62)$ & $38(80,85)$ & $1,09(0,72 ; 1,65)$ & \\
\hline > 1 salário mínimo & & $4(15,38)$ & $9(19,15)$ & 1,00 & \\
\hline Inicio do pré-natal & & & & & \\
\hline $1^{\circ}$ trimestre & & $18(69,2)$ & $24(51)$ & 1,00 & \\
\hline $2^{\circ}$ trimestre & & $8(30,8)$ & $22(46,8)$ & $1,28(0,92 ; 1,80)$ & 0,152 \\
\hline $3^{\circ}$ trimestre & & $0(-)$ & $1(2,13)$ & $1,75(1,34 ; 2,28)$ & $<0,001$ \\
\hline Classificação IMC pré-gestacional & & & & & \\
\hline Desnutrição & & $11(42,3)$ & $18(38,3)$ & 1,00 & \\
\hline Eutrofia & & $13(50)$ & $24(51,7)$ & $1,05(0,72 ; 1,52)$ & 0,817 \\
\hline Sobrepeso & & $1(3,9)$ & $3(6,4)$ & $1,21(0,64 ; 2,29)$ & 0,561 \\
\hline Obesidade & & $1(3,9)$ & $2(4,3)$ & $1,07(0,46 ; 2,53)$ & 0,870 \\
\hline Classificação IMC* & & & & & \\
\hline Desnutrição & & $5(19,23)$ & $15(31,9)$ & 1,00 & \\
\hline Eutrofia & & $16(61.5)$ & $26(55,3)$ & $0,83(0,58 ; 1,17)$ & 0,282 \\
\hline Sobrepeso & & $5(19,2)$ & $6(12,8)$ & $0,73(0,40 ; 1,33)$ & 0,298 \\
\hline Obesidade & & - & - & - & \\
\hline $\begin{array}{l}\text { *de acordo com a idade gestacional. IMC } \\
95 \% \text {. }\end{array}$ & ce de con & ção corpor & azão de pre & ência; IC 95\%: Intervalo & e confiança d \\
\hline $\begin{array}{l}\text { Tabela IV - Análise ajustada da assoc } \\
\text { e de saúde das adolescentes gestante }\end{array}$ & $\begin{array}{l}\text { entre oc } \\
\text { adidas em }\end{array}$ & $\begin{array}{l}\text { nsumo de fr } \\
\text { Im serviço } p\end{array}$ & $\begin{array}{l}\text { verduras e lę } \\
\text { o de referênc }\end{array}$ & $\begin{array}{l}\text { mes e características so } \\
\text { pré-natal. São Luís, M }\end{array}$ & $\begin{array}{l}\text { oeconômica } \\
\text { anhão, } 2015\end{array}$ \\
\hline & & Consumo & & & \\
\hline Variáveis & $\begin{array}{l}\text { adequado } \\
\text { n (\%) }\end{array}$ & in & $\begin{array}{l}\text { uado } \\
\text { o) }\end{array}$ & RP (IC 95\%) & p-valor \\
\hline Início do pré-natal & & & & & \\
\hline $1^{\circ}$ trimestre & $18(69,2)$ & & & 1,00 & \\
\hline $2^{\circ}$ trimestre & $8(30,8)$ & & $6,8)$ & $1,31(0,94 ; 1,83)$ & 0,109 \\
\hline $3^{\circ}$ trimestre & $0(-)$ & & & $1,61(1,23 ; 2,09)$ & $<0,001$ \\
\hline Escolaridade & & & & & \\
\hline$\geq 8$ anos de estudo & $16(61,5)$ & & $0,9)$ & 1,00 & 0,111 \\
\hline$<8$ anos & $10(38,5)$ & & ,1) & $0,66(0,40 ; 1,09)$ & \\
\hline
\end{tabular}

RP: Razão de prevalência; IC 95\%: Intervalo de confiança de $95 \%$. 


\section{DISCUSSÃO}

Verificou-se que a idade da amostra do presente estudo variou entre 14 e 19 anos, com média de 16,7 anos $\pm 1,3$ anos. Esta média encontrada diverge um pouco dos demais estudos. Uma pesquisa realizada no Paraná ${ }^{(17)}$ verificando o consumo alimentar de gestantes adolescentes de uma unidade básica de saúde observou uma média de 17,4 anos. O mesmo aconteceu com relação ao estudo realizado em São Paulo ${ }^{(18)}$, onde a média foi de 17,8 anos.

Percebeu-se uma precocidade de uniões conjugais das mães adolescentes, um dado relevante visto que mais de $50 \%$ eram casadas ou viviam em união consensual. Em uma pesquisa feita com grávidas adolescentes no Pará ${ }^{(19)}, \mathrm{o}$ percentual das adolescentes em união estável foi de 58,2\%. Em outro estudo ${ }^{(20)}$, realizado em São Paulo, apontou-se que $60 \%$ das adolescentes eram casadas ou viviam em união consensual. Dados próximos aos do presente estudo.

A gestação precoce pode se tornar mais um fator que contribui para o atraso escolar das jovens, considerando que muitas abandonam a escola após a ocorrência da gravidez ${ }^{(19)}$. No entanto, a escolaridade da maioria das adolescentes do presente estudo foi maior que 8 anos. Essa variável tem sido apontada como capaz de interferir na forma como a população escolhe seus alimentos, podendo ser decisiva para a qualidade do autocuidado e para a capacidade de interpretar informações relativas à proteção da saúde ${ }^{(21)}$.

Desta forma, acredita-se que a educação escolar é capaz de influenciar o conhecimento sobre alimentação e nutrição ${ }^{(22)}$ que, por sua vez, relaciona-se ao estado nutricional dos indivíduos ${ }^{(23)}$. Assim, indivíduos menos escolarizados demonstraram menor conhecimento sobre alimentação saudável. Isso sugere que poucos anos de estudo podem fazer diferença no entendimento sobre saúde e alimentação(24).

Outro fator relevante observado foi a instabilidade financeira observada em $71,2 \%$ das gestantes, que apresentaram renda familiar de no máximo 3 salários mínimos. Um estudo que avaliou o consumo alimentar de gestantes adolescentes na cidade de Fortaleza também encontrou uma maior concentração $(51,5 \%)$ de renda total das famílias na faixa de um a três salários mínimos. Além disso, $60,3 \%$ da amostra convivia com 4 ou mais membros na casa ${ }^{(25)}$. A dependência financeira absoluta da família ou do pai da criança gera maior risco de instabilidade conjugal, impossibilidade de estabelecer uma família com plena autonomia, autogestão e projeto de futuro ${ }^{(26)}$.

A renda familiar superior a um salário mínimo relatado pelas adolescentes não foi associada com consumo adequado de frutas, verduras e legumes. Observou-se que a renda menor (menos de um salário mínimo) teve um percentual de adequação maior. Em um estudo visando analisar a evolução da frequência declarada de consumo de carne bovina e de hortaliças no Brasil, observou-se que somente o aumento da renda dos consumidores não se traduz automaticamente em uma maior busca por uma alimentação mais saudável, ou seja, o aumento no consumo de verduras. Para que os consumidores busquem uma alimentação mais saudável estes devem possuir, além de renda elevada, um elevado nível de escolaridade ${ }^{(27)}$.

Dentre as alterações de estado nutricional observadas, a desnutrição foi mais prevalente, sendo observada em $39,7 \%$ das gestantes no período pré-gestacional e $27,4 \%$ no período gestacional. Este dado é preocupante, uma vez que poderá desencadear maior risco de retardo de crescimento intrauterino, mortalidade perinatal, doenças maternas, como diabetes gestacional, e dificuldades no parto ${ }^{(28)}$.

Em um estudo realizado na Colômbia para avaliação do estado nutricional de gestantes adolescentes, evidenciou um percentual de baixo peso de $44,7 \%{ }^{(29)}$. Outro estudo, realizado no Pará, com adolescentes grávidas internadas em um hospital de referência, os valores de baixo peso encontrados foram bem parecidos com os do presente estudo, $41,7 \%^{(19)}$.

Todas as pesquisas que avaliam o estado nutricional de gestantes adolescentes reforçam a importância de um acompanhamento nutricional individualizado durante a gestação. Ressalta-se que, com a avaliação do estado nutricional da gestante durante as consultas de pré-natal, é possível estabelecer as necessidades de nutrientes nesse período e direcionar as orientações nutricionais conforme cada diagnóstico.

Com relação ao consumo alimentar, os dados mostram um consumo muito frequente de alimentos de origem animal, destacando o leite e a carne bovina, e de outros alimentos tais como farinha, açúcar e salgadinho entre as gestantes adolescentes. $\mathrm{Na}$ análise do consumo diário dos alimentos, encontrou-se que os alimentos básicos, como arroz, açúcar, margarina e pão foram referidos por $75 \%$ ou mais das entrevistadas. Foi menos expressivo o consumo de verduras, legumes e frutas (laranja), sendo referido por cerca de $30 \%$ das jovens.

Esses resultados foram coerentes com as mudanças referidas no padrão da alimentação da população brasileira urbana nos últimos anos, destacando-se o aumento no consumo de carnes, de laticínios, de açúcar refinado e a diminuição de legumes, verduras e frutas na $\operatorname{dieta}^{(30)}$.

O consumo de frutas, verduras e legumes desse estudo foi mais adequado quando a gestante relatava união estável. Fato esse que pode ser justificado pela presença e o apoio do parceiro durante a gestação conferir maior segurança à adolescente e diminuição dos riscos de aborto durante o período da gestação ${ }^{(26)}$. 
A pouca frequência, verificada no consumo de frutas, verduras e legumes também foi verificada em outras duas pesquisas que avaliaramo consumo alimentar de adolescentes gestantes. Em decorrência dessas inadequações, ressaltouse a possibilidade de carência de vitaminas, principalmente A e $\mathrm{C}^{(25,3)}$. O ácido retinóico desempenha papel importante no período embrionário, atuando mais especificamente no desenvolvimento do coração, olhos e dos ouvidos ${ }^{(31)}$. A deficiência de vitamina $\mathrm{C}$, em gestantes, é relacionada ao surgimento de DHEG (doença hipertensiva específica da gravidez) e pré-eclampsia ${ }^{(4)}$.

Uma dieta pobre em frutas e vegetais e, ao mesmo tempo, rica em gorduras, pode estar relacionada ao aumento do risco de abortos espontâneos ou mesmo ser fator determinante desse risco $^{(32)}$.

Com relação a variável início do pré-natal, observouse que, quanto antes o pré-natal é iniciado, maiores são as chances das adolescentes terem uma alimentação adequada. As gestantes que iniciaram o pré-natal no $3^{\circ}$ trimestre têm $61 \%$ de chance de terem um consumo inadequado de frutas, verduras e legumes, quando comparadas àquelas que iniciam o pré-natal no $1^{\circ}$ trimestre de gestação.

$\mathrm{O}$ início precoce da assistência pré-natal permite o acesso aos métodos diagnósticos e terapêuticos para diversas patologias com repercussões graves para a saúde da mulher e do bebê, tais como hipertensão arterial crônica, diabetes não gestacional, anemia, infecção pela sífilis e pelo HIV. Além disso, propicia uma estimativa da idade gestacional mais precisa, com melhor monitoramento do crescimento fetal e melhor embasamento para decisões relacionadas a uma possível interrupção da gravidez ${ }^{(33,34)}$.

$\mathrm{Na}$ literatura, são escassas as informações a respeito da associação entre alimentação de adolescentes e o início do pré-natal. No entanto, observa-se que o pré-natal pode ser um meio de orientação e reeducação alimentar que proporcione um melhor entendimento da necessidade de uma alimentação saudável.

Não foi possível estabelecer uma relação causal entre o consumo de frutas, verduras e legume e fatores associados em gestantes adolescentes por se tratar de um estudo transversal, mas se pode inferir uma associação entre essas condições.

Assim, ressalta-se a necessidade de ações educativas com abordagem da situação socioeconômica e dos hábitos alimentares para auxiliar essas adolescentes na seleção de alimentos compatíveis com seu estado fisiológico. Destacase, também, o papel que cumpre a assistência pré-natal na aquisição de hábitos alimentares adequados à gestação, ainda neste ciclo de vida, que podem ficar incorporados à vida.

\section{CONCLUSÃO}

A maior parte das gestantes avaliadas apresentou-se eutrófica, apesar de cerca de um quarto apresentar baixo peso durante a gestação. Além disso, elas não consumiam uma dieta balanceada, com uma ingestão abaixo do recomendado de FVL. Entre os fatores relacionados a um melhor consumo de FVL destaca-se o início do acompanhamento pré-natal no primeiro trimestre.

\section{REFERÊNCIAS}

1. Meincke SMK, Oliveira MRP, Trigueiro DRSG, Carraro TE, Gondim ETC, Collet N. Perfil socioeconômico e demográfico de puérperas adolescentes. Cogitare Enferm. 2011;16(3):486-91.

2. Pedro F Filho, Sigrist RMS, Souza LL, Cunha D, Rassam ME. Perfil epidemiológico da grávida adolescente no município de Jundiaí e sua evolução em trinta anos. Adolesc Saúde. 2011;8(1):21-7.

3. Barros DC, Pereira RA, Gama SGN, Leal MC. O consumo alimentar de gestantes adolescentes no Município do Rio de Janeiro. Cad Saúde Pública. 2004;20(Supl 1):121-9.

4. Gomes RNS, Gomes VTS, Caldas DRC, Lago EC, Campos FKL, Gomes MS. Avaliação do estado nutricional de gestantes atendidas em unidades básicas de saúde de Caxias/MA. Rev Interdisciplinar (Teresina). 2014;7(4):81-90.

5. Story M, Moe J. Eating behaviors and nutritional implications. In: Story M, Stang J. Nutrition and the pregnant adolescent: a practical reference guide. Minneapolis: School of Public Health/University of Minnesota; 2000. p. 47-54.

6. Mourão JCA, Malaguti C, Moreira LPD, Faria NC, Rezende W, Rondelli RR, et al. Perfil alimentar e sociodemográficos de gestantes adolescentes da cidade de São Paulo. Rev Extendere. 2016;4(1):85-93.

7. Camargo RMS, Veiga GV. Ingestão e hábitos alimentares de adolescentes gestantes. Folha Médica. 2000;119(3):37-46.

8. Costa LCF, Vasconcelos FAG, Corso ACT. Fatores associados ao consumo adequado de frutas e hortaliças em escolares de Santa Catarina, Brasil. Cad Saúde Pública. 2012;28(6):1133-42.

9. Ministério da Saúde (BR). Programa de prevenção da gravidez precoce. Brasília: Ministério da Saúde; 2003. 
10. Muniz LC, Zanini RDV, Schneider BC, Tassitano RM, Feitosa WMDN, González-Chica DA. Prevalência e fatores associados ao consumo de frutas, legumes e verduras entre adolescentes de escolas públicas de Caruaru, PE. Ciênc Saude Coletiva. 2013;18(2):393404.

11. Freitas ES, Bosco SMD, Sippel CA, Lazzaretti RK. Recomendações nutricionais na gestação. Destaques Acadêmicos (Lajeado). 2011;2(3):81-95.

12. Sichieri R. Consultoria para desenvolvimento de um questionário simplificado de consumo alimentar. Rio de Janeiro: Universidade do Estado do Rio de Janeiro; 1998.

13. Sociedade Brasileira de Pediatria. Manual de orientação para a alimentação do lactente, do préescolar, do escolar, do adolescente e na escola. $3^{\mathrm{a}}$ ed. Rio de Janeiro: Departamento de Nutrologia; 2012.

14. Diniz LEV. Nutrição e gravidez. In: Zugaib BM, Sancrovski M. O pré-natal. Rio de Janeiro: Atheneu; 1994. p. 71-6.

15. Kominiarek MA, Rajan P. Nutrition recommendations in pregnancy and lactation. Med Clin North Am. 2016;100(6):1199-215.

16. Frisancho AR. Reduction of birth weight among infants born to adolescents: maternal-fetal growth competition. In: Jacobson MS. Adolescent nutritional disorders: prevention and treatment. New York: NewYork Academy of Sciences; 1997. p. 273-80.

17. Montovanelli L, Auler F. Consumo alimentar de gestantes adolescentes cadastradas na Unidade básica de saúde de Mandaguaçi, PR. Saude Pesqui (Impr). 2008;2(3):349-55.

18. Barros MAR, Nicolau AIO. Fatores nutricionais maternos e repercussões no peso do recém nascido. Rev Enferm UFPI. 2014; 3(2):49-55.

19. Moraes LP, Moraes PMO, Ribeiro ECD. Perfil epidemiológico e nutricional de adolescentes grávidas internadas em um hospital de referência do estado do Pará. Rev Para Medi. 2014;28(4):49-56.

20. Caminha NO, Brasil CC, Gomes RF, Sousa DMN, Freitas LV, Damasceno AKC. O perfil de puérperas adolescentes atendidas em uma maternidade de referência de Fortaleza-Ceará. Anna Nery Rev Enferm. 2012;16(3):486-92.

21. Velásquez-Meléndez G, Pimenta AM, Kac G. Epidemiologia do sobrepeso e da obesidade e seus fatores determinantes em Belo Horizonte (MG), Brasil: estudo transversal de base populacional. Rer Panam Salud Publica. 2004;16(5):308-14.

22. Quesada K, Bordin BC, Melo EV, Nascimento LMG, Mazzi NP. May obesity and nutritional knowledge influence the assessing energy intake underreporting in adults? Br J Med Med Res. 2016;12(3):1-8.

23. Dattilo M, Furlanetto P, Kuroda AP, Nicastro H, Coimbra PCFC, Simony RF. Conhecimento nutricional e sua associação com o índice de massa corporal. Nutrire Rev Soc Bras Aliment Nutr. 2009;34(1):75-84.

24. Lima TM, Osório MM. Breast-feeding: profile and associated factors in children under 25 months of age in Northeast Brazil. Rev Bras Saúde Matern Infant. 2003;3(3):305-14.

25. Azevedo DV, Sampaio HC. Consumo alimentar de gestantes adolescentes atendidas em serviço de assistência pré-natal. Rev Nutr. 2003;16(3):273-80.

26. Costa MCO, Santos CAST, Sobrinho CN, Moura MSQ, Souza KEP, Assis DR. Gravidez na adolescência: Associação de variáveis sociodemográficas e biomédicas materna com resultado neonatal. Rev Baiana Saúde Pública. 2014;29(2):300-12.

27. Novaes AL, Sproesser RL. Efeito do nível de escolaridade no consumo de carne bovina e hortaliças no Brasil. Campo Grande: UFMS; 2006.

28. Martins MLB, Tonial SR, Gama MEA, Silva THR, Ribeiro JM, Barbosa JMA. Consumo de alimentos entre adolescentes de um estado do Nordeste brasileiro. Demetra. 2014;9(2):577-94.

29. Marques CMF, Vieira FN, Barroso TMG. Adolescência no contexto da escola e da família - uma reflexão. Fam Saúde Desenvolv. 2003;5(2):141-6.

30. Tabares RQ, Astudilo MNM, Sierra LEA, Perea GAM. Estado nutricional y seguridad alimentaria en gestantes adolescentes. Pereira, Colombia. Invest Educ Enferm. 2010;28(2):204-13.

31. Monteiro CA, Mondini L, Costa RBL. Mudanças na composição e adequação nutricional da dieta familiar nas áreas metropolitanas do Brasil entre 1988-1996. In: Monteiro CA. Velhos e novos males da saúde no Brasil: a evolução do país e de suas doenças. $2^{\mathrm{a}}$ ed. São Paulo: Hucitec/NUPENS-USP; 2000. p. 359-70.

32. Vanucchi H, Amarelho PG. Vitamina A. In: Cozzolino SMF. Biodisponibilidade de nutrientes. $5^{\mathrm{a}}$ ed. Barueri: Manole; 2016.

33. Di Cintio E, Parazzini F, Chatenoud L, Surace M, Bensi G, Zanconato G. Dietary factors and risk of 
spontaneous abortion. Eur J Obstet Gynecol Reprod Biol. 2001;95(1):132-6.

34. Domingues RMSM, Viellas EF, Dias MAB, Torres JA, Theme-Filha MM, Gama SGN, et al. Adequação da assistência pré-natal segundo as características maternas no Brasil. Rev Panam Salud Publica. 2015;37(3):140-7.
Endereço para correspondência

Antônia Caroline Diniz Brito

Rua Cel. Eurípedes Bezerra, 100 Cond. Solar da Ilha 3, bloco 7 ap. 203

Bairro: Turú

CEP: 65066-260 - São Luís - MA - Brasil

E-mail: caroline turismo86@hotmail.com 